\title{
A puzzling bilateral diabetic foot problem
}

\author{
N Denecker ${ }^{1}$, D Aerden ${ }^{2}$, M De Maeseneer ${ }^{3}$
}

Diabetic foot clinic ${ }^{1.2}$ \& Radiology department ${ }^{3}$, Vrije Universiteit Brussel (VUB), Brussels, Belgium.

\section{Background}

Charcot neuroartropathy $(\mathrm{CN})$ of the foot is a rare but debilitating disorder that affects bones, joints and soft tissues of the foot and ankle. It is mainly seen as a late complication of diabetes neuropathy and can lead to significant deformity of the foot unless diagnosis is established early.

We report an unusual case of bilateral synchronous $\mathrm{CN}$ that proved particularly challenging because the diagnosis was obfuscated 1) by bilatera presentation of symptoms and 2) a preceding erysipelas. In addition, we had no prior experience in off-loading both limbs simultaneously.

\section{Case presentation}

A 58-year old woman with insulin dependent type 2 diabetes presented to the emergency department with fever and erythema of the left leg. The patient was on gabapentin for lower limb neuropathy and simvastatin for hypercholesterolemia. The left limb was erythematous and warm with a plantar neuropathic ulcer on the left hallux. Palpable distal pulses were present bilaterally. Blood sampling showed an important inflammatory reaction (Table 1). The diagnosis of erysipelas was established with the toe ulcer as entry point. A wound smear revealed Pseudomonas aeruginosa for which intravenous antibiotics were administered for 8 days.

Twelve days after discharge she returned with increased oedema and pain of the left leg, although inflammatory blood parameters had normalised. Local wound care was provided and the patient was seen 10 days later at the diabetic foot clinic. Inflammatory symptoms had persisted and had spread to the contralateral right foot and ankle: both feet were swollen, red and warm, and some bruises from a recent trauma were detected. X-rays of both feet were normal. A bone scintigraphy with SPECT-CT (Single Photon Emission Computed Tomography) was suggestive for $\mathrm{CN}$ of both feet, with uptake of tracer in both the left and right midfoot (Fig 1a). In addition, the CT revealed the presence of small bony fragments (Fig $1 \mathrm{~b}$ ). Hotspots over the 2 nd metatarsal heads bilaterally raised the possibility of underlying osteomyelitis. Inflammatory blood parameters had risen again.

Bilateral immobilization with total contact casts (TCC) was deemed impracticable. Hence, the left foot was treated with a removable air-cushioned cast (Aircast ${ }^{\circledR}$ ) instead and the patient was shortly hospitalized to provide basic comfort. Magnetic resonance imaging (MRI) was performed to exclude osteomyelitis: oedema of the tarsus and the metatarsal bases confirmed bilateral CN (Fig 2a) but osteomyelitis of the 2nd metatarsal head could only be rejected by a leucocyte scan with SPECT-CT. The patient was transferred to a rehabilitation centre and scheduled for regular follow-up at the diabetic foot clinic to renew the TCC. Three months later, clinical inflammatory signs and oedema of the midfoot on control MRI had significantly decreased, although increase of oedema was observed at the talar bone bilaterally (Fig 2b). The next 3 months, off-loading was accomplished with bilateral Aircast $₫$ walkers until custom made orthopaedics shoes became available with satisfactory ambulatory rehabilitation.

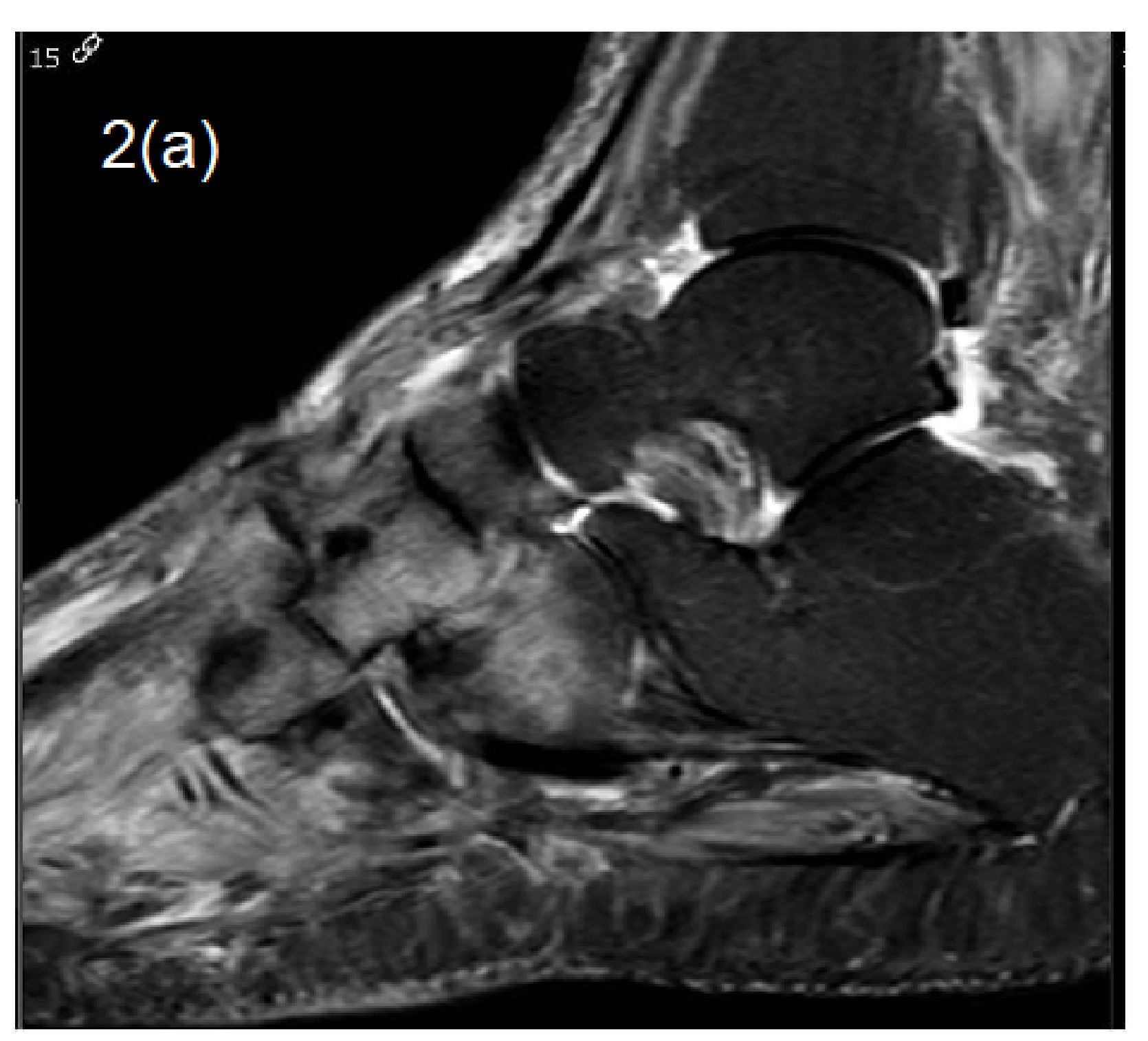

Figure 2(a): MRI of the right foot initially shows bone marrow oedema of the tarsus and metatarsal bases.

Figure 2(b): Three months later oedema in the original regions has improved but is now more prominent in the talar bone.
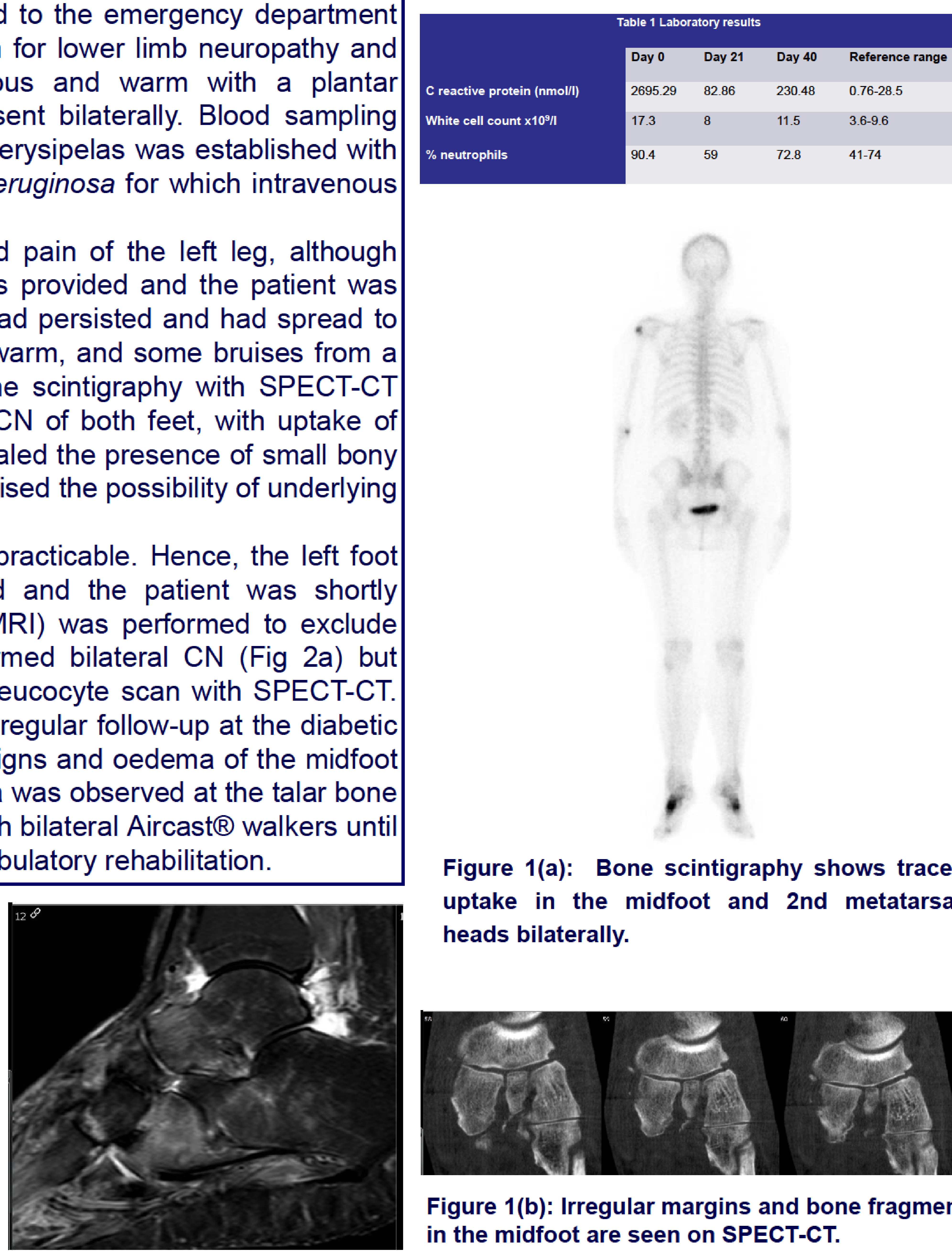

Figure 1(a): Bone scintigraphy shows trace uptake in the midfoot and 2 nd metatarsal heads bilaterally.

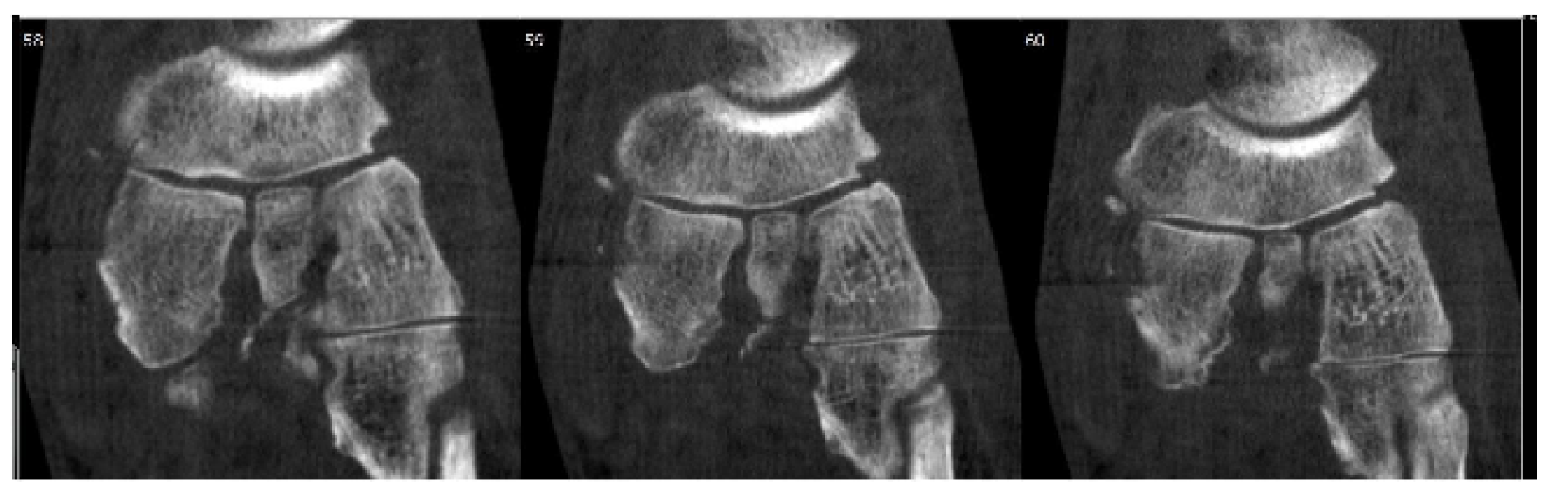

Figure 1(b): Irregular margins and bone fragments in the midfoot are seen on SPECT-CT.

\section{Discussion}

- $\mathrm{CN}$ is associated with an enhanced inflammatory response, presumably triggered by minor trauma, prior infection or ulceration, or foot surgery. Proinflammatory cytokines (TNF- $\alpha, \mathrm{IL}-1 \beta$ ) are released and lead to increased expression of the receptor activator of nuclear factor-KB (RANK) ligand, thereby activating NF-KB (Nuclear Factor KB), a potent promotor of osteoclastic activity which promotes osteolysis and fractures.

- The true prevalence and incidence of $\mathrm{CN}$ is unknown but is estimated to affect less than $1 \%$ of all patients with diabetes. Also the pathogenesis contributes to its rarity as $\mathrm{CN}$ will not occur if local inflammation is inhibited by a limited arterial inflow of the foot. Ipsilateral recurrence of $\mathrm{CN}$ is rare and contralateral $\mathrm{CN}$ may occur in $20 \%$ to $30 \%$ over several years.

- Diagnosis of acute Charcot foot is difficult because no specific laboratory tests are available, hence the diagnosis is primarily established clinically (a unilateral red, warm, swollen and often painless foot). Differential diagnosis should be made with infection (cellulitis, osteomyelitis, arthritis, abscess), acute gout, deep venous thrombosis and trauma (sprain, fracture). Imaging techniques are helpful but X-rays lack sensitivity during the first weeks. The sensitivity of bone scintigraphy is superior and its low specificity is improved by SPECT-CT. MRI has diagnostic accuracy in the early stages and allows differentiation from osteomyelitis. According to literature, the diagnosis of $\mathrm{CN}$ may be missed in $79 \%$ and delayed up to 29 weeks.

- Rapid immobilisation of the affected foot is paramount and is accomplished best by TCC, the gold standard for off-loading. A removable pneumatic walker achieves comparable off-loading but non-compliance remains a problem. Immobilisation is advised until clinical signs have resolved and a temperature difference of $<2^{\circ} \mathrm{C}$ between feet is recorded. In general, this occurs after 3-12 months, with 6 months being most common.

Conclusions and learning points

$>$ Synchronous bilateral involvement of both feet by Charcot neuroartropathy is extremely rare.

$>$ Triggers for Charcot neuroartropathy are miscellaneous and vary from infection to trauma, which complicates early diagnosis

> Simultaneous off-loading and immobilisation of both feet is challenging and can be managed by total contact cast and a removable pneumatic walker, which might necessitate admission in a rehabilitation centre.

\section{References}

Rogers LC, Frykberg RG, Armstrong DG, Boulton AJM, Edmonds M, Van Ha et al. The Charcot Foot in Diabetes. Diabetes Care 2011; 34(9): 2123-2129.

Gouveri E, Papanas N. Charcot osteoarthropathy in diabetes: A brief review with an emphasis on clinical practice. World J Diabetes 2011; 2(5): 59-65.

Botek G, Anderson MA, Taylor R. Charcot neuroarthropathy: An often overlooked complication of diabetes. Cleve Clin J Med 2010; 77(9): 593-599.

Pinzur MS. Current concepts review: Charcot arthropathy of the foot and ankle. Foot Ankle Int 2007; 28(8):952-9.

Jeffcoate WJ. Theories concerning the pathogenesis of the acute charcot foot suggest future therapy. Curr Diab Rep 2005; 5(6): 430-435.

Christensen TM, Gade-Rasmussen B, Pedersen LW, Hommel E, Holstein PE, Svendsen OL. Duration of off-loading and recurrence rate in Charcot osteo-arthropathy treated with less restrictive regimen with removable walker. J Diabetes Complications 2012; 26(5): 430-434.

Milne TE, Rogers JR, Kinnear EM, Martin HV, Lazzarini PA, Quinton TR et al. Developing an evidence-based clinical pathway for the assessment, diagnosis and management of acute Charcot Neuro-Arthropathy: a systematic review. J Foot Ankle Res 2013; 6(1): 30. 